\title{
Analytical Models of Optical Coherence Tomography for Tissue Optical Property Estimation: Preliminary Result and Comparison
}

\author{
Yan Wang, Jinming Duan, Li Bai \\ School of Computer Science, University of Nottingham \\ Nottingham, NG8 1BB, UK \\ yan.wang@nottingham.ac.uk, bai@cs.nott.ac.uk
}

\begin{abstract}
Optical Coherence Tomography (OCT) can provide non-invasive imaging of living tissues based on the principle of optical interferometry. Though there is an abundant amount of literature on OCT image processing and segmentation, recovering optical parameters of the biological tissue from OCT data remains a challenge and demands further research, as tissue optical properties play an important role in disease diagnosis. In this paper we consider estimating tissue optical properties from OCT data as an inverse problem. We will review the main approaches for the forward step of the inverse problem to generate the OCT signal using both the extended Huygens-Fresnel principle (EHF), which is a theoretical model for OCT imaging based on optics, and the Radiative Transfer Equation (RTE), which describes mathematically the energy transfer through a media. Our experimental results show a clear agreement between these two models for OCT modelling.
\end{abstract}

Keywords: Optical Coherence Tomography, Inverse Problem, Extended Huygens-Fresnel Principle, Radiative Transfer Function.

\section{Introduction}

Optical Coherence Tomography (OCT) is a non-invasive imaging technique that can capture optical properties of biological tissues such as scattering and absorption properties. Changes in tissue micro-structure due to disease would alter these optical properties, estimating tissue optical properties is thus important for a wide range of applications including medical diagnosis, tissue engineering, and developmental biology. These optical properties are however invisible in OCT images so conventional image analysis algorithms are not suitable to detect them. Machine learning could be used to map tissue optical properties to the OCT signal so that tissue optical properties can be estimated from OCT data. However, machine learning methods to learn the mapping often require a considerable amount of training data that is representative of the mapping to be learnt, and this is often not available.

In this paper we consider OCT image analysis as an inverse problem. An inverse problem generally involves two steps, that is, in the context of OCT, a forward step, which synthesises the OCT signal given tissue parameters, and an inverse step, which minimises the difference between the real OCT signal and the synthesised one. We will first review the main approaches for forward modelling of the OCT signal (Section 2). We will then provide details in (Section 3) for implementing the Extended Huygens-Fresnel principle (EHF) and the Radiative Transfer Equation (RTE) models for OCT modelling. The RTE model has been used for modelling optical tomography which is however different from OCT in principle, since it does not involve low-coherence interferometry. We present in Section 4 our experimental results for comparison of the RTE and the EHF models for OCT modelling, followed by the conclusion in Section 5.

\section{The OCT Modelling Literature in Brief}

The principle behind OCT is low-coherence interferometry [5]. To show how OCT could be used to analyse tissue optical properties we show a diagram of a generic OCT system in Fig. 1. Put simply, a light beam from a light source is split into a reference arm and a sample arm. The light exiting the sample arm is guided through a fibre and focused onto the sample tissue to be examined. The light through the reference arm is reflected back from a mirror, thus experiencing a time delay. The backscattered light from the tissue is redirected back to the system through the same fibre and combined with the returning light from the reference arm. The mixed light creates an interference pattern on the surface of a photoreceiver or detector, which is then converted into electronic signals. The signals are processed into a reflectivity profile of the sample 
tissue at various scan depths and this is called the A-scan. As the sample arm sweeps laterally across the tissue sample, many A-scans are generated along the lateral direction and are combined into a cross-sectional 2D image, namely, the B-scan. Bscans resulted from different lateral directions are combined into a 3D volume dataset.

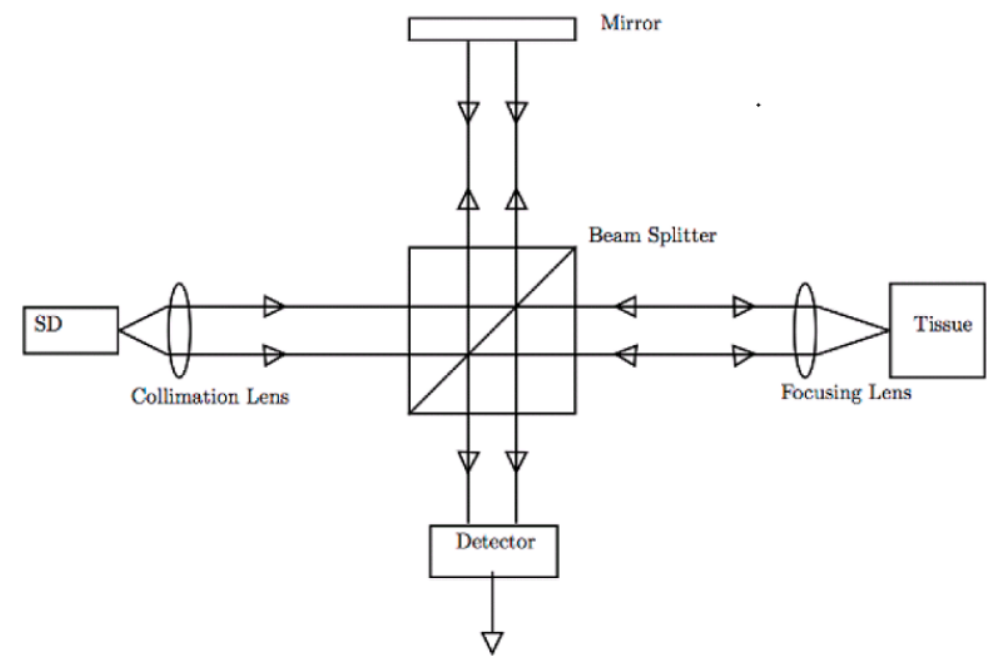

Fig. 1: A diagram of a generic OCT system. SD: superluminescent diode.

There are in general two major approaches for modelling the OCT imaging process. The first approach uses Monte Carlo (MC) simulation based on an understanding of how light is propagated in scattering media such as biological tissues. The second approach is the analytical approach that uses equations derived from the theory of optics or energy transfer, such as the Maxwell's equation, and the Extended Huygens-Fresnel principle (EHF) [1].

MC based OCT modelling [3] simulates photon and tissue interaction during light transport within the scattering media. Given the geometry and optical coefficients of a medium and characteristics of a light beam, a large quantity of simulated photons are tracked while propagating through a medium which are subject to stochastic processes of absorption, reflection and scattering along their paths. Main advantages of MC based models include its applicability to tissues with complex geometry or optical properties and its flexibility for incorporating different optical phenomena by simulating specific photontissue interaction. However, a major drawback of the approach is that the computation for solving the inverse problem can be very costly, since a large number of photons have to be traced at each update of the parameters to be estimated. Examples of MC based models include the MCML simulator [9] for studying the propagation of a laser beam incident into multilayered tissues. The tissue is assumed homogeneous and isotropic. The TIM-OS simulator [6] was based on MCML and allows for simulation of photon interaction in inhomogeneous media with varying refractive index. The media is modelled with a tetrahedron-based finite element mesh. Photons may be reflected or refracted while going through a face of the tetrahedron subject to a probability, as neighbouring tetrahedrons may have different refractive index. The MC based simulator described in [4] incorporates the coherence length of the light and different types of photons in the formation of OCT signals.

Examples of analytical models based on the Maxwell's equation and EHF can be found in [7] for wave propagation through a non-absorbing media featuring spatial varying refractive index and multiple scattering events. The model is used to estimate the scattering coefficient $\mu_{s}$ and the effective anisotropy factor $g_{\text {eff }}$ of both single-layered and multi-layered media. It does not consider the absorption coefficient $\mu_{a}$ since its magnitude is generally negligible compared to $\mu_{s}$ for tissues concerned. A simplified Maxwell's equation is as follows:

$$
\Delta^{2} U(\vec{R})+k^{2} n^{2}(\vec{R}) U(\vec{R})=0
$$

where $U(\vec{R})$ is the scalar component of the electric field of the propagating wave which is transverse to the propagating direction at a position $\vec{R}$, and $k$ is the wavenumber and $n(\vec{R})$ is the refractive index at $\vec{R}$ which contains random fluctuation around a mean index. 
Eq. (1) is a stochastic equation and the EHF is used to obtain an approximate solution as follows. Let $\vec{p}$ be a 2D position at the detector. Let $U_{R}(\vec{p})$ be the reference field at $\vec{p}$ and $U_{S}(\vec{p} ; z)$ be the sample field at $\vec{p}$ reflected from the tissue at a probed depth $z$. By mixing both fields at the detector, the mean square heterodyne signal current $\left\langle i^{2}(z)\right\rangle$ generated for the probed depth $z$ is given as:

$$
\left\langle i^{2}(z)\right\rangle=2 \alpha^{2} \operatorname{Re}\left(\iint \Gamma_{S}\left(\vec{p}_{1}, \vec{p}_{2} ; z\right) \Gamma_{R}\left(\vec{p}_{1}, \vec{p}_{2}\right) d \vec{p}_{1} d \vec{p}_{2}\right)
$$

where Re means taking the real part of a complex number, $\Gamma_{R}\left(\vec{p}_{1}, \vec{p}_{2}\right)=U_{R}\left(\vec{p}_{1}\right) U_{R}^{*}\left(\vec{p}_{2}\right)$, the mutual coherence function of the reference field, and $\Gamma_{S}\left(\vec{p}_{1}, \vec{p}_{2} ; z\right)=\left\langle U_{S}\left(\vec{p}_{1} ; z\right) U_{S}^{*}\left(\vec{p}_{2} ; z\right)\right\rangle$, the averaged mutual coherence function of the sample field over the statistical properties of the tissue. \$lalpha $\$$ is the power to current conversion factor.

In contrast, the ISOCT model [10] was a single scattering model, which assumes that the medium has continuous refractive index (RI) fluctuations. However, single scattering models are generally not suitable for biological tissues as when photons propagate to a certain depth through tissues with varying refraction index (i.e., inhomogeneous), a considerable number of them are highly likely to be scattered multiple times. Without considering multiple scattering, errors can occur in optical parameter estimation.

Finally, the OCT model described in [8] was based on the Radiative Transfer Equation (RTE) under the small angle approximation. The RTE describes mathematically the phenomenon of energy transfer in the form of electromagnetic radiation through a medium affected by emission, absorption, and scattering processes. The RTE has been used for modelling optical tomography which is however different from OCT in principle, since it does not involve low-coherence interferometry. In this paper we will provide details of our experiments using both EHF and RTE for OCT forward modelling and validate RTE as a suitable technique for OCT modelling.

\section{Forward OCT Modelling}

\subsection{Using EHF}

\subsubsection{Single-Layered Tissue Model}

Let $\mu_{s}$ and $g$ be the scattering coefficient and anisotropy factor of the simulated tissue, which is assumed to have a refractive index $n$. The settings of the OCT system are as follows: the sample arm light beam with a radius $w_{0}$ is focused onto the surface of the tissue by a focusing lens with a focus length $f$, so that the distance from the lens to the tissue surface, $d$, is equal to $f$. Let $k$ be the wavenumber of the probing light beam: $k=2 \pi / \lambda$, where $\lambda$ is the wavelength. The mean square heterodyne signal current $\left\langle i^{2}(z)\right\rangle$ is formulated as

$$
\left\langle i^{2}(z)\right\rangle=\left\langle i^{2}(z)\right\rangle_{0} \Psi(z)
$$

where $\left\langle i^{2}(z)\right\rangle_{0}$ is the mean square heterodyne signal current in the absence of scattering at depth $z, \Psi(z)$ is the heterodyne efficiency factor representing signal degradation due to scattering. $\left\langle i^{2}(z)\right\rangle_{0}$ is calculated as

$$
\left\langle i^{2}(z)\right\rangle_{0}=\frac{\alpha^{2} P_{R} P_{S} \sigma_{b}}{\pi w_{H}^{2}(z)}
$$

where $\alpha$ is the power to current conversion factor, $P_{R}$ and $P_{S}$ are the power of the reference and the sample arm beams respectively, $\sigma_{b}$ is the effective backscattering cross-section, and $w_{H}^{2}(z)$ is the $1 / e$ radius of the irradiance at the probing depth $z$ defined as

$$
w_{H}^{2}(z)=w_{0}^{2}\left(A-\frac{B(z)}{f}\right)^{2}+\left(\frac{B(z)}{k w_{0}}\right)^{2}
$$

where $A$ and $B(z)$ are entries of the ABCD ray-matrix for light propagation from the lens plane to the probing depth. For this case where the sample arm light beam is focused on the surface of the tissue, we have $A=1$ and $B=d+z / n$. $\Psi$ is expressed as 


$$
\Psi(z)=\exp \left(-2 \mu_{s} z\right)+\frac{4 \exp \left(-\mu_{s} z\right)\left[1-\exp \left(-\mu_{s} z\right)\right]}{1+w_{S}^{2}(z) / w_{H}^{2}(z)}+\left[1-\exp \left(-\mu_{s} z\right)\right]^{2} \frac{w_{H}^{2}(z)}{w_{S}^{2}(z)}
$$

Here $w_{S}^{2}(z)$ is the $1 / e$ irradiance radius of the light beam at depth $z$ with presented scattering, which is formulized as

$$
w_{S}^{2}(z)=w_{H}^{2}(z)+\left(\frac{2 B(z)}{k \rho_{0}(z)}\right)^{2}
$$

where $\rho_{0}(z)$ is the lateral coherence length defined as follows

$$
\rho_{0}(z)=\sqrt{\frac{3}{\mu_{s} z}} \frac{\lambda}{\pi \theta_{r m s}}\left(\frac{n B(z)}{z}\right)=\sqrt{\frac{3}{\mu_{s} z}} \frac{\lambda}{\pi \theta_{r m s}}\left(1+\frac{n d}{z}\right)
$$

where $\theta_{r m s}$ is the root-mean-square scattering angle.

Eq. (6) contains three items: the first item represents the component of the probing light undergoing single scattering, the third item is the component experiencing multiple scattering due to tissue inhomogeneity and the second item is the coherence mixing of the unscattered and multiple scattered light. The anisotropy factor fitted with this model is the effective anisotropy factor, $g_{e f f}=\cos \theta_{r m s}$. The item $n d / z$ in Eq. (8) represents the shower curtain effect, since the lateral coherence length increases as the distance between the lens and the tissue surface increases, which leads to degeneration of the OCT image resolution. It is worth noting that $\mu_{s}$ and $\theta_{r m s}$ in Eq. (4) are sufficient to determine the shape of the OCT signal. Specifically, in case of a dynamic-focusing OCT system (i.e., the focusing plane is coincidental with the tissue plane at the probing depth while the lens is moving longitudinally during A-scan), $\left\langle i^{2}(z)\right\rangle_{0}$ is a constant proportional to $\sigma_{b}$ since $w_{H}^{2}(z)$ is constant w.r.t. $z$.

\subsubsection{Multi-Layered Tissue Model}

The single layered EHF model described in [7] can be extended to multi-layered tissues. Let $N$ be the number of layers in the tissue. $z_{n}$ is the probing depth within the $n$-th layer. The $i$-th layer $(i=1,2, \cdots, N)$ has a refractive index $n_{i}$, a scattering coefficient $\mu_{s i}$, a root-mean-squared scattering angle $\theta_{r m s, i}$ and a depth $\Delta_{i}$. The heterodyne efficient factor is similar to Eq. (3), except the following modification to incorporate optical coefficients of multiple layers

$$
\begin{gathered}
z=d+\sum_{i=1}^{n-1} \Delta_{i}+z_{n} \\
\mu(z)=\sum_{i=1}^{n-1} \mu_{s i} \Delta_{i}+\mu_{s n}\left(z_{n}\right)
\end{gathered}
$$

We replace $\mu_{S} z$ in Eq. (6) with $\mu(z)$ which is the total optical path length of the probing beam within the tissue. Define $l_{n-1}=d+\sum_{i=1}^{n-1} \Delta_{i}$, the distance between the lens plane and the $n$-th layer. The definition of $w_{H}$ and $w_{S}$ is the same as the single-layer model, but the lateral coherence length needs to be revised as

$$
\rho_{0}(z)=\frac{\lambda \sqrt{3} B(z)}{\pi \sqrt{\sum_{i=1}^{n} \theta_{r m s, i}^{2} \mu_{s i} n_{i}\left(B_{b}^{3}\left(l_{i}\right)-B_{b}^{3}\left(l_{i+1}\right)\right)}}
$$

where $=d+\sum_{i=1}^{n-1} \frac{\Delta_{i}}{n_{i}}+\frac{z_{n}}{n_{n}}, B_{b}\left(l_{i}\right)=\sum_{j=i}^{n} \frac{\Delta_{j}}{n_{j}}$ and $B_{b}\left(l_{n+1}\right)=0$. 


\subsection{Using RTE}

Our aim is to compare RTE and EHF for OCT modelling. Let $L(z, \boldsymbol{\rho}, \boldsymbol{n}) d V d \boldsymbol{n}$ be the intensity of radiation in a volume element $d V$ centred at a point $(z, \boldsymbol{\rho})$ defined in a cylindrical coordinate system where $z$ is along the direction of radiation and $\boldsymbol{\rho}$ is a transverse $2 \mathrm{D}$ vector, and travelling within a solid angle element $d \boldsymbol{n}$ centred around the direction $\boldsymbol{n}$. The RTE is given as

$$
\left(\boldsymbol{n} \cdot \nabla+\mu_{s}(z)+\mu_{a}(z)\right) L(z, \boldsymbol{\rho}, \boldsymbol{n})=\frac{\mu_{s}(z)}{4 \pi} \int x\left(z, \boldsymbol{n} \cdot \boldsymbol{n}^{\prime}\right) L\left(z, \boldsymbol{\rho}, \boldsymbol{n}^{\prime}\right) d \boldsymbol{n}^{\prime}
$$

where $x\left(z, \boldsymbol{n} \cdot \boldsymbol{n}^{\prime}\right)$ is the scattering phase function at the depth $z \cdot \mu_{s}(z)$ and $\mu_{a}(z)$ are the scattering and absorption coefficients respectively, which need to be estimated. If we assume that almost all photons travel along the direction of radiation (i.e., the $z$ axis), that is, the angle that the path of the photons makes with the $z$ axis should be very close to zero, we will have $\boldsymbol{n} \approx\left(1, \boldsymbol{n}_{\perp}\right)$ and $\boldsymbol{n} \cdot \boldsymbol{n}^{\prime} \approx\left|\boldsymbol{n}_{\perp}-\boldsymbol{n}_{\perp}^{\prime}\right|$. We then obtain the small angle approximation of RTE as follows

$$
\left(\frac{\partial}{\partial z}+\boldsymbol{n}_{\perp} \cdot \nabla_{\perp}+\mu_{s}(z)+\mu_{a}(z)\right) L\left(z, \boldsymbol{\rho}, \boldsymbol{n}_{\perp}\right)=\frac{\mu_{s}(z)}{4 \pi} \int x\left(z,\left|\boldsymbol{n}_{\perp}-\boldsymbol{n}_{\perp}^{\prime}\right|\right) L\left(z, \boldsymbol{\rho}, \boldsymbol{n}_{\perp}^{\prime}\right) d \boldsymbol{n}_{\perp}^{\prime}
$$

Given the boundary condition, this equation can be solved either numerically or analytically with a proper choice of the scattering phase function. In [8], for OCT, the scattering phase function is defined as a sum of a small-angle scattering phase function and a constant corresponding to isotropic scattering.

$$
x(z, \gamma)=\left(1-2 p_{b}(z)\right) x_{1}(z, \gamma)+2 p_{b}(z)
$$

Here $\gamma$ is the scattering angle and $p_{b}$ is the backscattering probability to be estimated. $x_{1}(z, \gamma)$ is defined as a Gaussian density function

$$
x_{1}(z, \gamma)=\frac{4}{\left\langle\gamma^{2}(z)\right\rangle} \exp \left(-\frac{\gamma^{2}}{\left\langle\gamma^{2}(z)\right\rangle}\right)
$$

where $\left\langle\gamma^{2}(z)\right\rangle$ is the variance of the scattering angle at depth $z$ to be estimated.

Let $U^{U P S}(z, \boldsymbol{\rho})$ be the radiance of a unidirectional point light source at a position $(z, \boldsymbol{\rho})$ due to radiation propagation of the probing light beam. It can be calculated as integration of the radiation intensity over all solid angles as follows

$$
U^{U P S}(z, \boldsymbol{\rho})=\int_{4 \pi} L\left(z, \boldsymbol{\rho}, \boldsymbol{n}_{\perp}\right) d \boldsymbol{n}_{\perp}^{\prime}
$$

The probing beam is a distributed light source, modelled as a 2D Gaussian distribution:

$$
\mathrm{A}(z, \boldsymbol{\rho})=\frac{P_{S} \exp \left(-\|\boldsymbol{\rho}\|^{2} / a^{2}(z)\right)}{\pi a^{2}(z)}
$$

where $a^{2}(z)=a_{0}^{2}+\left((z-f) \lambda / 2 \pi n a_{0}\right)^{2}$, the spot size at $z, a_{0}$ is the spot size at the focal plane and $\lambda$ is the wavelength, $n$ is the mean refractive index of the medium, and $P_{S}$ is the power of the probing beam.

Since the probing beam is a distributed light source, the light source at $(z, \boldsymbol{\rho})$ is also distributed and its radiance can be calculated as

$$
U(z, \boldsymbol{\rho})=A(z, \boldsymbol{\rho})^{\circ} U^{U P S}(z, \boldsymbol{\rho})
$$

where ${ }^{\circ}$ is the convolution operator. 
The amount of backscattered light is thus calculated as $x(z, \pi) \mu_{s}(z) U(z, \boldsymbol{\rho})$. The OCT signal obtained from the heterodyne detector is calculated as the integration of backscattered energy over the detector aperture accounting for correlations between the forward propagating light and the backscattered light, i.e.,

$$
I(z)=2 p_{b}(z) \mu_{s}(z) B \int_{-\infty}^{\infty}\left(2 U^{2}(z, \boldsymbol{\rho})-U_{0}^{2}(z, \boldsymbol{\rho})\right) d \boldsymbol{\rho}
$$

where $U_{0}^{2}(z, \boldsymbol{\rho})$ is the radiance of the (unscattered) ballistic component of the light,

$$
U_{0}^{2}(z, \boldsymbol{\rho})=A(z, \boldsymbol{\rho}) \exp \left(-\int_{0}^{z}\left(\mu_{s}\left(z^{\prime}\right)+\mu_{a}\left(z^{\prime}\right)\right) d z^{\prime}\right)
$$

and $B$ is a constant determined by a specific OCT system.

\section{Results \& Comparison}

To simulate the OCT signal, we use a numerical RTE multigrid solver [2]. This method was originally proposed to solve general RTEs and the original scattering phase function is the Henyey-Greenstein (HG) scattering phase function. We replaced the GH phase function by the small angle scattering phase function defined in Eq. (14). Since we seek a numerical solution, the small approximation of RTE is not necessary. After the radiance function is obtained by solving the RTE equation, Eq. (19) is evaluated numerically to construct the final OCT signals. The OCT setup uses wavelength $1300 \mathrm{~nm}$, focal length $0.5 \mathrm{~mm}$, beam waist radius $7.5 \mu \mathrm{m}$ [8]. Besides, we set the absorption coefficient to zero, the refractive index to 1.38 (valid for many biological tissues) and the lens-to-tissue distance to $0.3 \mathrm{~mm}$.

We simulated two two-layered model tissues with parameters presented in Table 1 and 2 . The dimension of both tissues is set to $0.2(\mathrm{~L}) \times 0.2(\mathrm{~W}) \times 1.0(\mathrm{D}) \mathrm{mm}$. We use default parameter settings for the RTE multigrid solver

Table 1: Parameters of the first two-layered model tissue.

\begin{tabular}{|l|l|l|l|l|}
\hline Layer & depth $(\mathrm{mm})$ & $\mu_{s}, \mathrm{~mm}^{-1}$ & $p_{b}$ & $\left\langle\gamma^{2}\right\rangle$ \\
\hline 1 & 0.2 & 5 & 0.07 & 0.4 \\
2 & 0.8 & 10 & 0.2 & 0.1 \\
\hline
\end{tabular}

Table 2: Parameters of the second two-layered model tissue.

\begin{tabular}{|l|l|l|l|l|}
\hline Layer & depth $(\mathrm{mm})$ & $\mu_{s}, \mathrm{~mm}^{-1}$ & $p_{b}$ & $\left\langle\gamma^{2}\right\rangle$ \\
\hline 1 & 0.3 & 1.7 & 0.043 & 0.17 \\
2 & 0.7 & 6.1 & 0.14 & 0.12 \\
\hline
\end{tabular}




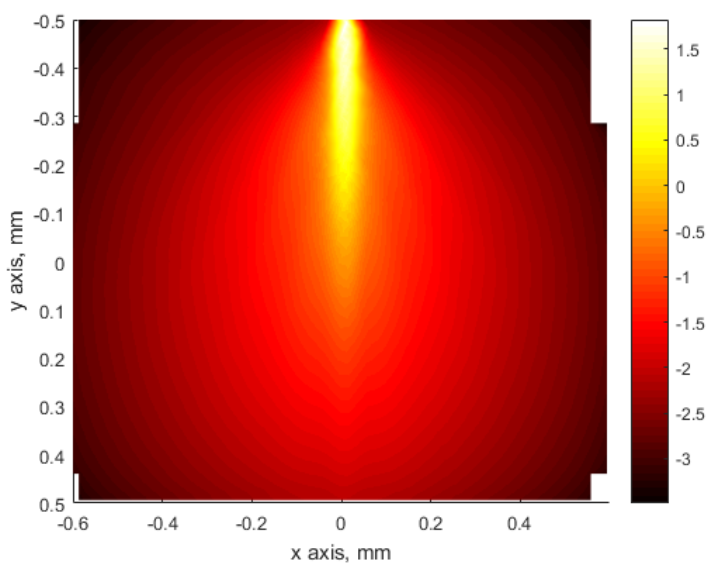

(a)

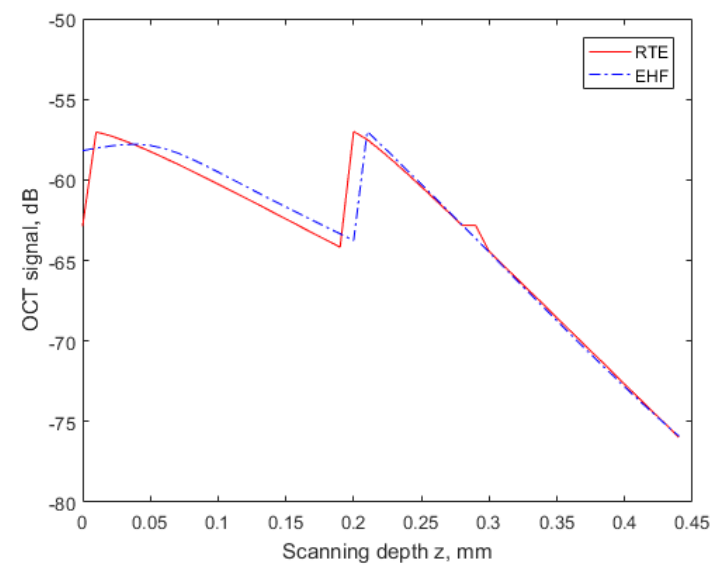

(c)

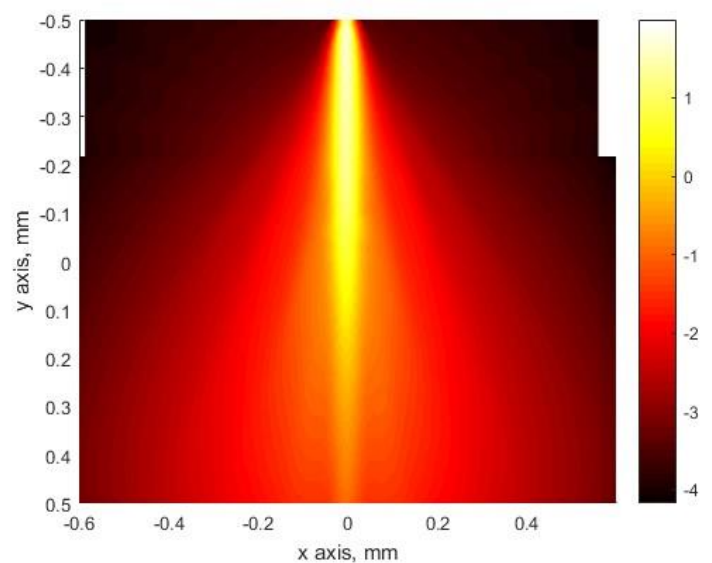

(b)

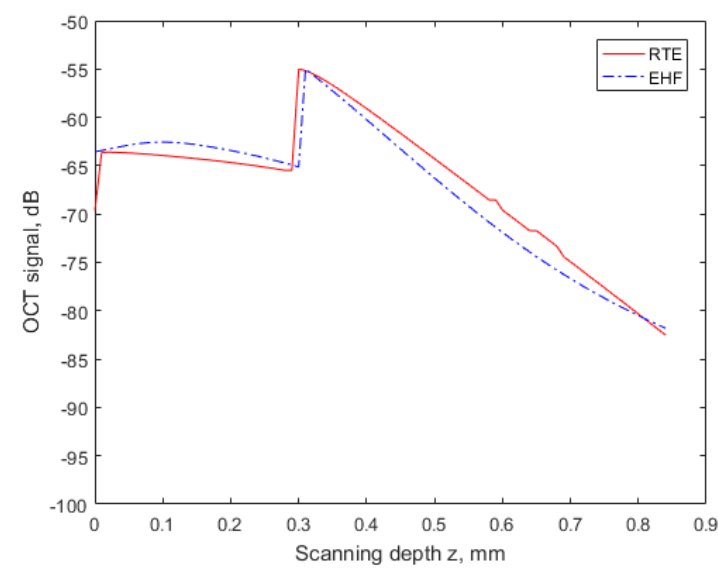

(d)

Fig. 2: Simulation result using two-layered tissue model defined in [8]. (a) and (b) show the light radiance function as the solution to the RTE resulted from the propagation of a unidirectional point light source placed at the top of the model tissues. (c) and (d) are the simulated OCT A-scan using both the RTE and EHF models.

The shape of the tissue model is defined in three dimensions. The numerical solver requires triangulation of the shape of the tissue model into tetrahedrons. The simulation results are presented in Fig. 2. Note that the result is expressed using the logarithmic decibel scale $(\mathrm{dB})$. The overall shape of the simulated OCT signals appears consistent with Fig. 5 in [8]. We also simulated the OCT signals with the EHF model under the same settings, as shown in Fig. 2(c) and (d) as comparison. The simulation results using both models are largely consistent. As the EHF principle is known to model OCT signals well, the comparison result validates the RTE model for OCT modelling. The magnitude of the simulated signal is determined by $B$ in Eq. (19) and the power of the probing beam, $P_{S}$ in Eq. (18). However, the magnitude is irrelevant, since the geometric and optical parameters are sufficient to determine the shape of the OCT signal. For the inverse problem, the simulated OCT signal should be fitted to the real signal up to a constant multiplier and, equivalently, up to a transverse shift in the logarithmic decibel scale. We also simulated a B-scan of a model tissue with two layers. The second layer has a curved shape. The B-scan image is shown in Fig. 3. 


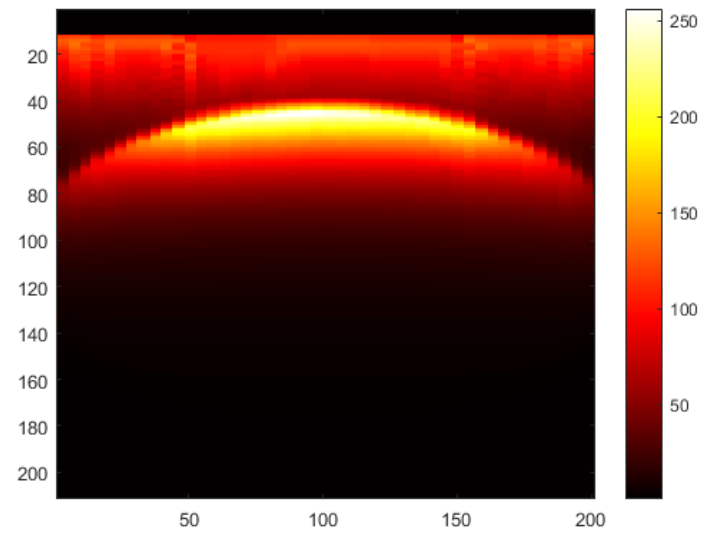

Fig. 3: A simulated B-scan image of a two-layered model tissue. Resolution $201 \times 211$ pixels of length $5 \mu m$.

\section{Conclusion}

In this paper, we reviewed state of the art techniques for modelling the OCT signal. We have implemented both EHF and RTE models for the modelling and demonstrated their similar results. Our next step will be to tackle the inverse step of the inverse problem to estimate tissue optical parameters from the OCT signal. Solving the inverse problem typically employs an optimisation process, which can lead to ill-posed inverse problems for complex tissue structures, that is, there may be no unique solutions to the inverse problem. We will however benefit from the advances in solutions to inverse problems in the mathematical and geophysical research communities for our OCT inverse problem to estimate tissue optical properties for applications.

\section{References}

[1] P. E. Andersen, T. M. Jørgensen, L. Thrane, A. Tycho, H. T. Yura, "Modelling Light-Tissue Interaction in Optical Coherence Tomography Systems," Optical Coherence Tomography, Springer, Berlin, Heidelberg, 2008.

[2] H. Gao and H. Zhao, "A fast forward solver of radiative transfer equation," Transport Theory and Statistical Physics, vol. 38, pp. 149-192, 2009.

[3] M. Kirillin, I. Meglinski, V. Kuzmin, E. Sergeeva, and R. Myllylä, "Simulation of optical coherence tomography images by Monte Carlo modelling based on polarization vector approach," Optics Express, vol. 18, no. 21, pp. 21714 21724, 2010.

[4] S. Malektaji, I. T. Jr Lima, S. S. Sherif, "Monte Carlo simulation of optical coherence tomography for turbid media with arbitrary spatial distributions," J. Bio. Opt., vol. 19, no. 4, 2014.

[5] J. A. Izatt and M. A. Choma, "Theory of Optical Coherence Tomography," Biological and Medical Physics, Biomedical Engineering, Springer, Berlin, Heidelberg, 2008.

[6] H. Shen and G. Wang, "A tetrahedron-based inhomogeneous Monte Carlo Optical Simulator," Physics in Medicine and Biology, vol. 55, no. 4, pp. 947-962, 2010.

[7] L. Thrane, M. H. Frosz, D. Levitz, P. E. Andersen, "Extraction of tissue optical properties from optical coherence tomography images for diagnostic purposes," in Proceedings of SPIE - The International Society for Optical Engineering, 2005.

[8] I. V. Turchin, E. A. Sergeeva, L. S. Dolin, V. A. Kamensky, N. M. Shakhova, R. Richards-Kortum, "Novel algorithm of processing optical coherence tomography images for differentiation of biological tissue pathologies," Journal of Biomedical Optics, vol. 10, no. 6, 2005.

[9] L. Wang, S. L. Jacques, L. Zheng, "MCML-Monte Carlo Modelling of Light Transport in Multi-layered Tissues," Computer Methods and Programs in Biomedicine, vol. 47, pp. 131-146, 1995.

[10] J. Yi, A. J. Radosevich, J. D. Rogers, S. C. P. Norris, İ. R. Çapoğlu, A. Taflove, and V. Backman, "Can OCT be sensitive to nanoscale structural alterations in biological tissue?," Optics Express, vol. 21, pp. 9043-9059, 2013. 